\title{
CALIDAD DE EMPLEO CON ENFOQUE DE GÉNERO: PROPUESTA DE UN MARCO CONCEPTUAL
}

Claudia Jessenia Becerra Gualdrón

claudia.becerra@uptc.edu.co

Universidad Pedagógica y Tecnológica de Colombia (Colombia)

Recibido: 08-04-2014

Aceptado: 19-04-2015

\section{Resumen}

Las mujeres se encuentran en peores condiciones que los hombres en la mayoría de esferas de la vida. Además de esto, ciencias como la economía, con enfoque androcéntrico, invisibilizan la problemática. El análisis de la calidad de vida laboral (CVL) con enfoque de género tiene un abordaje escaso, por esta razón, en el presente artículo se presenta un marco conceptual para su análisis del cual surgen dos instrumentos de medición, uno para los trabajadores y otro para la empresa. Estos instrumentos se basan, tanto en percepciones subjetivas como en datos objetivos, los cuales permiten por un lado, analizar la CVL dentro de una empresa y por otro, comparar los niveles con enfoque de género.

Palabras clave: Calidad de empleo, género, marco conceptual,

\begin{abstract}
Women are in worse conditions than men in most spheres of life. Besides, sciences, like economics, with an androcentric approach, ignore the problem. The analysis of the quality of working life (QWL) with a gender approach is poor; for these reasons, in this article, a conceptual framework for analysis is presented, emerging two measuring instruments, one for workers and one for the company. These instruments are based both on subjective perceptions and objective data, which allow, on the one hand, to analyze the QWL within a company and secondly, to compare levels with gender.
\end{abstract}

Keywords: Quality of working life, gender, background. 


\section{Introducción}

Los cambios acaecidos en las formas de organización productiva, las reformas laborales, las dinámicas de integración comercial y los procesos de globalización han hecho que se preste una especial atención a la calidad de vida laboral. Además, en la actualidad, el éxito de una organización está estrechamente vinculado con la forma en que atrae, motiva y retiene su fuerza laboral; lo cual depende de su capacidad de brindar una calidad alta de vida laboral (Aziz et al, 2011). En tal sentido, es posible afirmar que el análisis de la calidad de vida laboral (CVL) es importante desde diversos enfoques y es deseable desde puntos de vista económicos y sociales.

Sin embargo, la CVL, así como muchos otros aspectos de la vida humana, no es compartida en igualdad de condiciones por hombres y mujeres. La preocupación por una mayor equidad entre géneros no es asunto nuevo, desde hace varios años, se viene trabajando por abordar los desafíos laborales desde un enfoque que conduzca a un desarrollo productivo con más equidad para América Latina y se resalta la importancia de la desagregación por sexo de la información estadística para la visibilización de las mujeres (Pautassi, 2007).

De esta forma, el presente documento pretende exponer el marco conceptual sobre el cual descansa el abordaje de la calidad de empleo y su enfoque en la diferenciación de género. El análisis se centra desde el punto de vista de la ciencia económica, sin perder de vista que la CVL incluye múltiples dimensiones. El artículo se estructura de la siguiente manera: en primer lugar se presenta evidencia de la desigualdad de género y el abordaje desde la economía, luego se trata la calidad de vida laboral, su contextualización, definición y sus principales características, asimismo, se extiende esta noción hacia el enfoque de género, posteriormente, se revisan las aproximaciones a metodologías de medición de la CVL y se presenta la propuesta de modelo de medición de la calidad de vida laboral incluyendo el enfoque de género; se finaliza con algunas reflexiones. 


\section{Desigualdad de género: Evidencia}

Las sociedades humanas han ido constituyendo sus propios sistemas de organización para garantizar su reproducción y supervivencia. En esta organización, dan jerarquías a sus integrantes en función del lugar que ocupan y las actividades que desempeñan. Una de las categorías más importantes y determinante en la organización social de los pueblos ha sido la división entre lo femenino y lo masculino, la cual tiene su nacimiento en las diferencias sexuales entre hombres y mujeres (Sanchís, 2005).

En ninguna sociedad, actualmente, las mujeres disfrutan de las mismas oportunidades que los hombres. Existe una amplia evidencia acerca de la situación de desventaja de las primeras frente a los últimos. Según el Informe sobre Desarrollo Humano de las Naciones Unidas (PNUD, 2011), a pesar de los extraordinarios avances que han tenido algunos países en la equidad de género, en otros existe una profunda inequidad.

En el mundo, persiste la mortalidad femenina en temas relacionados con el embarazo y el parto. En este sentido, se encuentra que en países como Australia y Cuba el $100 \%$ de las mujeres tienen al menos una visita prenatal y los partos son atendidos por personal de salud calificado; en países como Afganistán, Somalia y Etiopía, este porcentaje varía del 6 al 26\%, lo cual refleja la desatención y el alto riesgo para las mujeres y sus hijos. Para Colombia, el porcentaje de mujeres que tiene por lo menos una visita prenatal es de $94 \%$ y el $96 \%$ con atención calificada (PNUD, 2011).

Asimismo, en el mundo, por cada 100.000 nacimientos, en países como Afganistán mueren 1.400 mujeres, esta situación señala la gran vulnerabilidad en la cual se encuentran las mujeres por el simple hecho de serlo. Asimismo, la tasa de prevalencia del VIH es mayor para las mujeres que para los hombres.

Además, existe una fuerte brecha educacional entre hombres y mujeres. En el mundo, la tasa de alfabetización en hombres es de $88,3 \%$, mientras que la de las mujeres es de 79,2\% (Index Mundi, 2012). La brecha entre géneros de población que cuenta con al menos educación secundaria en países como Ghana supera el 49\% y en Togo el 29\% (PNUD, 2011).

En este mismo sentido, las mujeres siguen accediendo en su mayoría a carreras consideradas tradicionalmente femeninas, aunque poco a poco han ido incursionando en las ciencias de la naturaleza, el aumento es muy lento. En otras palabras, las estructuras sexistas que se reflejan principalmente en las áreas científicas y tecnológicas, siguen intactas (Queralt y 
Ruiz, 2003). De modo que la ciencia y la tecnología se han desarrollado en un contexto que ha excluido a la mujer (Solsona, 1996).

Por su parte, en el mercado laboral las mujeres también se ubican en situaciones desventajosas respecto de los varones: acceden a menos empleos, obtienen menos remuneraciones, y se encuentran más sometidas a la desprotección social (Rodríguez, 2010).

En todo el mundo existe una gran brecha salarial entre hombres y mujeres. En todos los países del mundo, los hombres ganan más que las mujeres por el mismo trabajo. Mujeres con igual o mejor condición educativa y de experiencia profesional son remuneradas peor que los hombres. Así por ejemplo Scanlan (2004), señala que las mujeres trabajan más horas que los hombres, sin embargo la mayoría de sus labores siguen siendo no remuneradas, no reconocidas y subvaloradas.

La diferencia entre la tasa de participación laboral de hombres y mujeres supera el 26\%, los países con mayor brecha son Paquistán (63,2\%) y Arabia Saudí $(58,6 \%)$, para Colombia, esta brecha asciende a 36,9\% (PNUD, 2011).

\subsection{Abordaje económico de la desigualdad de género}

\subsubsection{Clásicos y neoclásicos: Una visión androcéntrica}

A pesar de la anterior evidencia de desigualdad entre hombres y mujeres en casi todos los aspecto de la vida humana, existe una renuencia notable en la literatura estándar sobre el desarrollo económico a tratar la posición de las mujeres como un problema separado (Sen, 1987). La teoría económica es profundamente androcéntrica, desde el nacimiento de la economía como ciencia, la mujer ha tenido un papel secundario (Escribano y Pardo, 2010)

Siguiendo la revisión de Martínez (2011), se puede afirmar que la economía clásica afronta el problema económico desde un enfoque de reproducción. Un sistema económico enfrenta problemas para resolver el problema económico fundamental cuando uno o varios factores de la producción tienen problemas para reproducirse. Sus propuestas se basan en asegurar condiciones para que los factores de producción se reproduzcan en el tiempo.

Mientras tanto, la economía neoclásica aborda el problema económico desde un enfoque de asignación de los factores de producción. Un sistema económico enfrenta problemas para resolver el problema económico fundamental cuando uno o varios factores de la producción no son asignados de manera eficiente y eso provoca desequilibrios (micro, macro, o ambos). Sus 
propuestas son asegurar condiciones para una asignación eficiente de factores: Mercado libre, Intervención del Estado en el mercado, Regulaciones sobre el mercado y Creación y/o fortalecimiento de instituciones.

Un punto común tanto del enfoque clásico como neoclásico es su contenido androcéntrico, teniendo en cuenta que estos desarrollos teóricos fueron generados por hombres para interpretar la realidad económica desde las expectativas masculinas: Trabajadores, empresarios, terratenientes (Adam Smith, David Ricardo), Proletarios y Capitalistas (Carlos Marx) y para los neoclásicos Homo Ecomomicus (Martínez, 2011).

Una de las principales críticas de la economía actual es tratar a los agentes económicos como seres homogéneos (humano, varón, blanco, adulto, heterosexual, sano), racionales, plenamente informados y con iguales capacidades y oportunidades para elegir libremente (Martínez, 2011). Además, la economía está hecha entendiendo un matrimonio heterosexual con hijos en la que el hombre es ganador del pan y la mujer es ama de casa (Flacso, 2010). Por tanto, la economía neoclásica falla al incorporar las dimensiones de discriminación propias de las relaciones sociales incluidas las de género.

En suma, la teoría económica neoclásica considera algunos bienes como variables externas de la economía entregados gratuitamente por la naturaleza y por tanto no se incluyen en el cálculo económico (Guzmán y Todaro, 2002). De manera que por ejemplo la preparación de los alimentos o el cuidado de los niños o ancianos es una labor que no se tiene en cuenta para el cálculo económico y no se considera productiva.

\subsubsection{Análisis de la unidad familiar: una visión más amplia}

A pesar de lo anterior, desde la teoría económica si se han hecho esfuerzos por entender la interacción de hombres y mujeres y se ha considerado que una de las mejores formas de entender este problema es desde el análisis de la unidad familiar. Así por ejemplo, en la "Nueva economía del hogar", Becker (1987), considera la familia como una unidad armoniosa, en la cual existe un jefe altruista que representa la función de preferencias que maximiza la utilidad conjunta de los miembros del hogar.

Por otro lado, para Sen (1987), el papel de los sesgos en la percepción y los poderes de negociación, son los que explican las decisiones familiares con respecto a la inversión en capital humano y división del trabajo por género. Este trabajo es útil para entender por qué se 
presenta la inequidad y qué mecanismos se pueden utilizar para disminuirla, desde la argumentación de Conflictos Cooperativos.

Según esta teoría, todas las personas tienen diversas identidades (ser mujer u hombre, ocupación, nacionalidad, etc.). En algunos contextos la identidad familiar puede tener una fuerte influencia en la percepción de bienestar individual, en algunas sociedades tradicionales, las mujeres no conciben su bienestar individual separado de los miembros de la familia. El anterior se convierte en un problema de percepción, hay una inviabilidad de la noción de bienestar personal, lo cual, junto con una gran preocupación por el bienestar de la familia, ayuda a mantener la desigualdad tradicional. De modo que los marginados aceptan la legitimidad de la desigualdad, convirtiéndose en cómplices implícitos, con lo cual no hay protestas.

Para Sen (1987), aparte de la percepción existen aspectos objetivos para evaluar el bienestar de una persona: sus funcionamiento y capacidades (qué es capaz él o ella de hacer o de ser: la capacidad de sobrevivir, de mantenerse nutrido, de leer, escribir y comunicarse; de hacer parte de la vida de una comunidad).

Además, es posible diferenciar el "bienestar" del "agente". Mientras que hay un vínculo evidente entre los objetivos de una persona y su bienestar, el éxito como agente puede no estar conectado estrechamente -y ciertamente puede no estar identificado- con el bienestar de esa persona. El aspecto "agente" es el que está más influenciado por el sentido de la obligación de una persona y la percepción de la conducta legítima. Los grupos marginados pueden estar habituados a la inequidad y pueden estar dispuestos a aceptar la legitimidad del orden establecido.

Por otra parte, a pesar que la supervivencia y reproducción de los trabajadores es obviamente esencial, las actividades de sustento, supervivencia o reproducción normalmente no se tienen en cuenta como una contribución al producto y es clasificada como "labor improductiva".

En otro sentido, Sen afirma que la prosperidad de un hogar depende de varias actividades, una de ellas es la división del trabajo por género. Así, los miembros del hogar enfrentan dos problemas simultáneamente: la cooperación (suma de las disponibilidades totales) y el conflicto (división de las disponibilidades totales entre los miembros de la familia). Los contratos sociales, acerca de quién hace qué, quién consume qué, quién toma qué decisión; 
puede ser vista como respuesta a este problema de cooperación y conflicto. La división sexual de trabajo es uno de estos contratos sociales.

Desde el punto de vista de la tecnología y la producción, estos contratos sociales, ponen de manifiesto el trabajo gastado en actividades que no están definidas en la producción; así como la estabilidad y supervivencia de patrones de desigualdad y la profundamente asimétrica división sexual del trabajo; además de la división entre trabajo remunerado y no remunerado y el sesgo en la percepción de quién produce qué y quién gana qué, lo que es central para entender la posición económica inferior de las mujeres en las sociedades tradicionales (e incluso en las modernas). Finalmente, para entender los patrones de la división sexual del trabajo incluso fuera del hogar.

La simultaneidad del conflicto y la cooperación en las divisiones de género ha sido trivializada y evitada en muchos modelos económicos. Para Sen, una forma de ver este problema es a través de los “problemas de negociación”, la cooperación refleja los poderes de negociación de cada una de las partes. En el caso más simple, hay dos personas con intereses bien definidos que se percibe en la forma de dos funciones de utilidad cardinales. Se puede cooperar por completo. El resultado cuando no se puede cooperar puede ser llamado "la posición de status quo" o "el punto de quiebre".

Sen, utiliza tres "respuestas", relacionadas con "el punto de quiebre" (si el punto de quiebre de una persona fuera peor en términos de bienestar, entonces la solución colusiva, sería menos favorable a su bienestar), el "interés percibido" (si a la percepción de interés propia de una persona, se le atribuyera menos valor que el bienestar propio, entonces, la solución colusiva, sería menos favorable al bienestar de esa persona) y la "contribución percibida" (si en la contabilización de todas las contribuciones, se percibe que una persona está haciendo una contribución mayor a la opulencia del grupo, la solución colusiva, sería más favorable para esa persona), para esclarecer el sesgo entre los sexos. De este modo, por ejemplo, la maternidad y la crianza de los hijos, debe ser el resultado del conflicto cooperativo menos favorable para las mujeres, a través de un peor punto de quiebre y una menor habilidad para hacer contribuciones percibidas a la fortuna económica de la familia; también se pueden tener en cuenta otros factores como mayor analfatetismo y menor educación.

Los "ganadores", en una primera ronda de solución colusiva, tienen no sólo un resultado más satisfactorio, sino un mayor poder de negociación en el futuro y un mejoramiento de las habilidades para negociar en el futuro (para mejorar las bases de poder de la oferta que una 
persona puede llegar a tener en el futuro) (Ejemplo: tener una mejor educación, libertad para trabajar fuera del hogar). La transmisión también puede funcionar de generación en generación, como los patrones típicos de empleo y educación de hombres y mujeres. En el contexto actual, estas asimetrías, son estables y sostenidas, y la debilidad relativa de las mujeres en conflictos cooperativos en un período tiende a sostener la debilidad relativa en el próximo.

\section{Calidad de vida laboral (CVL)}

Un empleo puede ser definido como una actividad que genera una remuneración y se puede extender para incluir el trabajo voluntario y de no mercado (Aziz et al., 2011). En las actuales sociedades occidentales, se ha otorgado un papel muy importante al trabajo remunerado y es considerado como algo deseable en la búsqueda de la felicidad, que es, desde algunos puntos de vista el propósito de la vida (Gandelman y Piani, 2013) ya que además de generar un ingreso, facilita el progreso social y económico, y fortalece a las personas, a sus familias y comunidades (OIT, 2013).

El trabajo es una dimensión importante en las vidas de las personas. El desempleo involutantario tiene un gran impacto negativo no material sobre la felicidad y satisfacción de la gente (Clark y Oswald, 1994; Winkelmann y Winkelmann, 1998; Frey y Stutzer, 2001; Gandelman y Piani, 2013). Según el estudio de Gandelman y Piani (2013), la probabilidad de ser felices es mayor para los trabajadores que para los no trabajadores, debido, entre otras razones a factores como la mayor satisfacción con la vida económica y la salud.

El término calidad de empleo ha tenido una amplia aceptación en la literatura de diversas áreas como la economía, la antropología, la sociología y la psicología y afecta no solo los resultados organizacionales, sino la salud y el bienestar de los empleados (Pichler y Wallace, 2009). La promoción del empleo de alta calidad, ha sido aceptada como una prioridad para la sociedad, dadas sus implicaciones desde el punto de vista ético y en su importancia en el desarrollo de la humanidad (Spencer, 2012).

La calidad de empleo o CVL, es una noción que surgió durante los sesentas-setentas, cambiando el enfoque del bienestar de las personas en el trabajo (Ingelgard y Norrgren, 2001). El movimiento de la calidad de vida laboral, nace por un lado de la necesidad de "humanizar" los sistemas de producción basados en una fuerte división del trabajo, que tuvieron sus raíces en los modelos tayloristas los cuales buscaban reducir las labores a los componentes mínimos para aumentar la productividad del trabajo; por otro lado, en los 60, se popularizó el concepto 
"alienación" que buscaba estrategias alternativas para el diseño del trabajo y el empoderamiento de los trabajadores. Además, la "descualificación” o pérdida de destrezas que presentaban los trabajadores al realizar labores repetitivas y finalmente, los efectos físicos y mentales sobre las personas de calidad de vida laboral pobre; hicieron que fuera tomando fuerza el movimiento de calidad de vida laboral (Downing, Ryan, Mcneive, Mariani, y Parlangeli, 1995).

Actualmente, los desarrollos acerca de la CVL, se han enriquecido con la teoría de las necesidades de Maslow, la teoría de los dos factores de Herzberg, la teoría de las tres necesidades de McClleland y la teoría del efecto indirecto de Alderfer (1977); según las cuales, las necesidades básicas del individuo emergen de vez en cuando y hay algunos efectos indirectos en su vida personal y familiar (Noor y Abdullah, 2012). Además, en el debate internacional, la calidad del empleo y el trabajo decente ganaron importancia a partir de los años noventa, en especial por parte de instituciones como la Organización Internacional del Trabajo y Naciones Unidas (Weller y Roethlisberger, 2011).

El fundamento conceptual de la CVL, se encuentra enraizado en las discusiones sobre calidad de vida, bienestar y felicidad y se ha enfocado de diversas maneras que varían desde el disfrute subjetivo, la satisfacción de preferencias o el desarrollo de capacidades. Desde el enfoque de capacidades se asigna importancia a la capacidad de los individuos para decidir y optar por una vida mejor (Nussbaum y Sen, 1996). El trabajo y su contexto (arreglos y normas sociales, formales e informales) le proporciona a una persona, a través de los funcionamientos que obtiene de él (destrezas profesionales, atención en salud, relaciones personales, poder adquisitivo, estatus, etcétera), una serie de capacidades específicas de desempeño, relación, valoración y opción por distintos estilos de vida; es decir, distintos grados de libertad.

Teniendo en cuenta que la CVL tiene por objetivo determinar los aspectos del trabajo que inciden en la calidad de vida de una persona, se debe tener en cuenta el trabajo en sus múltiples expresiones en el ser y hacer de una persona que van más allá del salario, por tanto, se puede afirmar que es de carácter multidimensional, además, implica la reproducción de la sociedad, la construcción de identidad y sentido de pertenencia, la posibilidad de reconocimiento y satisfacción social (Pineda, 2006).

La retribución en dinero es el motivante principal para trabajar, para comer, pagar cuentas, tener donde vivir, etc. Sin embargo, el dinero no es el único motivo por el cual la gente trabaja, diversos estudios demuestran que las personas continúan trabajando a pesar de 
no tener necesidad económica de hacerlo, como los ganadores de loterías (Spector, 2008; Arvey, Harpaz y Liao, 2004); además, un empleo es una buena forma de crear y mantener relaciones interpersonales así como de satisfacer necesidades de autoestima y desarrollo personal (Galić y Plećaš, 2012).

Otra característica que se puede asignar a la calidad del empleo es que tiene elementos tanto subjetivos como objetivos de valoración (Pineda, 2006). Sin embargo, las subjetivas dependen de las preferencias y expectativas personales, de modo que una mayor satisfacción puede estar relacionada con menores expectativas

De acuerdo con lo revisado anteriormente, la CVL es tratada de diferentes maneras, como una variable, como una aproximación, como un conjunto de métodos o como un movimiento (Nadler y Lawlor, 1983). Aunque para algunos autores, existen diferencias entre los conceptos de CVL y trabajo de calidad, otros autores los toman como sinónimos (Galić y Plećaš, 2012), para los fines del presente documento, se tomarán como dos nociones con el mismo significado. En el cuadro 1, se presentan algunas nociones relacionads con la CVL a través del tiempo según diferentes autores.

Cuadro 1. Definiciones de Calidad de Vida Laboral

\begin{tabular}{|l|l|}
\hline Autor & Definición \\
\hline Walton (1973) & $\begin{array}{l}\text { Identificó ocho grandes categorías de importancia para el análisis de la } \\
\text { CVL: Compensación adecuada y justa, Medio ambiente seguro y } \\
\text { saludable, Desarrollo de las capacidades del ser humano, Crecimiento y } \\
\text { seguridad, Integración social, Constitucionalismo y derechos, Espacio } \\
\text { para la vida y Relevancia social en la vida. }\end{array}$ \\
\hline Suttle (1977: 4) & $\begin{array}{l}\text { "El grado en que los miembros de una organización de trabajo son } \\
\text { capaces de satisfacer necesidades personales importantes a través de sus } \\
\text { experiencias en la organización" }\end{array}$ \\
\hline $\begin{array}{l}\text { Straw } \\
\text { Heckscher (1984) }\end{array}$ & $\begin{array}{l}\text { CVL es una filosofía, un conjunto de principios, que sostiene que las } \\
\text { personas son el recurso más importante en la organización, ya que son } \\
\text { dignos de confianza, responsable y capaz de hacer valiosa contribución } \\
\text { y deben ser tratados con dignidad y respeto. }\end{array}$ \\
\hline $\begin{array}{l}\text { Delamotte } \\
\text { Takezawa (1984) }\end{array}$ & $\begin{array}{l}\text { La CVL abarca todos los aspectos de la vida laboral que posiblemente } \\
\text { podría ser de interés para la satisfacción del trabajador y la motivación. }\end{array}$ \\
\hline $\begin{array}{l}\text { Greenhaus, } \\
\text { Bedeian } \\
\text { Mossholder (1987) }\end{array}$ & $\begin{array}{l}\text { La CVL, está relacionada con la satisfacción de los empleados y los } \\
\text { comportamientos relacionados con el trabajo. }\end{array}$ \\
\hline $\begin{array}{l}\text { Cunningham } \\
\text { yom }\end{array}$ & \begin{tabular}{l} 
Los elementos que sean relevantes para la "calidad de la vida en el \\
\hline
\end{tabular}
\end{tabular}




\begin{tabular}{|c|c|}
\hline Eberle (1990) & $\begin{array}{l}\text { trabajo individual como el trabajo, el medio ambiente físico de trabajo, } \\
\text { el entorno social dentro de la organización, el sistema administrativo y la } \\
\text { relación entre la vida dentro y fuera del trabajo. }\end{array}$ \\
\hline Nicholson (1995) & $\begin{array}{l}\text { Hace referencia a las reacciones de los empleados para trabajar, sobre } \\
\text { todo los resultados personales relacionados con la satisfacción en el } \\
\text { trabajo, la salud mental y la seguridad. }\end{array}$ \\
\hline Sen (1999) & $\begin{array}{l}\text { Es un derecho que va más allá de la legislación laboral vigente, “.... que } \\
\text { tiene el sentido de reconocer derechos básicos y que permite una } \\
\text { comprensión mucho más alentadora de las necesidades de las distintas } \\
\text { instituciones y las distintas políticas en pro de los derechos y de los } \\
\text { intereses de los trabajadores" }\end{array}$ \\
\hline Saklani (2004) & $\begin{array}{l}\text { CVL es definida como la calidad de la experiencia humana como la } \\
\text { interacción de la relación empleado-organización. }\end{array}$ \\
\hline $\begin{array}{l}\text { Che Rose et al. } \\
(2006)\end{array}$ & $\begin{array}{l}\text { La CVL consta de oportunidades para la participación activa en la } \\
\text { organización del trabajo en grupo o la solución que sean de beneficio } \\
\text { mutuo con los empleados o empleadores, basado en la cooperación de } \\
\text { gestión laboral de problemas. La gente también conciben CVL como un } \\
\text { conjunto de métodos, tales como grupos de trabajo autónomos, } \\
\text { enriquecimiento laboral y alta participación destinado a impulsar la } \\
\text { satisfacción y productividad de los trabajadores }\end{array}$ \\
\hline Serey (2006) & $\begin{array}{l}\text { La definición tiene que ver con un trabajo significativo y satisfactorio. } \\
\text { Incluye (i) la oportunidad de ejercer unos talentos y capacidades, para } \\
\text { hacer frente a retos y situaciones que requieren una iniciativa } \\
\text { independiente y auto-dirección, (ii) una actividad que se cree que vale la } \\
\text { pena por las personas involucradas, (iii) una actividad en que se entiende } \\
\text { el papel que juega los objetivos individuales en el logro de algunos } \\
\text { objetivos generales, y (iv) una sensación de sentirse orgulloso de lo que } \\
\text { está haciendo y hacerlo bien. }\end{array}$ \\
\hline Pineda (2006: 29) & $\begin{array}{l}\text { "La CVL se puede definir como un fenómeno multidimensional desde } \\
\text { un marco teórico que la relaciona con el análisis de la calidad de vida, } \\
\text { una aproximación basada en el enfoque de las capacidades y el } \\
\text { desarrollo humano, y en las contribuciones que a ellos ha hecho el } \\
\text { pensamiento feminista" }\end{array}$ \\
\hline $\begin{array}{l}\text { Azziz et al. (2011: } \\
\text { 150) }\end{array}$ & $\begin{array}{l}\text { "La calidad de la relación entre trabajadores y el total del ambiente de } \\
\text { trabajo" }\end{array}$ \\
\hline $\begin{array}{lll}\text { Galić } & \text { y } & \text { Plećaš } \\
(\mathbf{2 0 1 2}) & & \end{array}$ & $\begin{array}{l}\text { Es un constructo multifacético, por lo general su definición y } \\
\text { componentes están basados en teorías psicológicas y de satisfacción de } \\
\text { necesidades, las cuales son buscadas por las personas en sus trabajos }\end{array}$ \\
\hline OIT (2012) & $\begin{array}{l}\text { El trabajo es fuente de dignidad personal, estabilidad familiar, paz en la } \\
\text { comunidad, democracias que actúan en beneficio de todos, y } \\
\text { crecimiento económico, que aumenta las oportunidades de trabajo } \\
\text { productivo y el desarrollo de las empresas. }\end{array}$ \\
\hline Spencer (2012) & $\begin{array}{l}\text { La CVL, implica un trabajo que paga bien, que ofrece un verdadero } \\
\text { grado de autonomía, y que presenta oportunidades para el desarrollo de } \\
\text { habilidades. }\end{array}$ \\
\hline
\end{tabular}

Fuente: Elaboración propia

Cuestiones de género: de la igualdad y la diferencia - e-I.S.S.N: 2444-0221 - No 10, 2015 - pp. 194-224 
De acuerdo con lo anterior, para efectos del presente documento, la calidad del empleo se tomará como una noción multidimensional, estrechamente ligada con el bienestar, la calidad de vida y aquello que una persona juzga que le es favorable para ella, que se genera de la relación del trabajo con la vida humana.

\section{CVL con enfoque de género}

La importancia de la desagregación por género de la CVL, nace de las grandes diferencias existentes entre hombres y mujeres tanto en la esfera laboral como en la calidad de vida en general. Otorgar un enfoque de género a la CVL, implica reconocer ciertos aspectos específicos, en este sentido, se analizarán cuales podrán ser las implicaciones de las siguientes dimensiones de la CVL según el género de las personas:

\subsection{CVL y enfoques subjetivos}

Según algunos autores, la CVL, tiene una gran carga de subjetividad (Delamotte y Takezawa, 1984; Greenhaus, Bedeian \& Mossholder, 1987; Saklani, 2004; Serey, 2006) sin embargo, una aproximación que intenta incorporar el enfoque de género al análisis de la CVL, debe tener mucho cuidado con la valoración de acuerdo a características subjetivas como las expectativas de los individuos ya que estos pueden incorporar sesgos bastante grandes.

En este sentido, la mayor satisfacción subjetiva reportada por algunas mujeres, no indica necesariamente que estén en una situación superior a la de los hombres sino se debe a su mejoramiento a situaciones extremas de opresión "El hecho de que una trabajadora haya aprendido a vivir en la adversidad de su trabajo y a sonreír ante ella, como a reconocer que posiblemente está mejor que su madre, no significa que su empleo sea de buena calidad" (Pineda, 2006:32).

Desde el enfoque de capacidades, como afirma Sen "puede ser un serio error tomar la ausencia de protesta y cuestionamiento de la inequidad como evidencia de la ausencia de tal inequidad" (1990: 126). Entonces, la valoración desde el enfoque de capacidades, propone no concentrarse en la reacción mental frente al empleo sino en las capacidades que este genera en la persona. 


\subsection{CVL y reproducción de la fuerza de trabajo}

Existe una marcada diferencia entre los llamados trabajos productivos y reproductivos. Aunque dentro de la literatura de las ciencias sociales el trabajo doméstico había recibido poco reconocimiento, a partir de la publicación de El origen de la familia, propiedad privada y el estado de Engels, se propuso una diferenciación entre el trabajo remunerado, con aporte directo y contabilizado a la economía conocido como trabajo "productivo" y el trabajo no remunerado, con aporte indirecto y no contabilizado a la economía realizado dentro de los hogares, conocido como trabajo "reproductivo" (Gammage y Orozco, 2008).

Las actividades reproductivas dentro del hogar se definen como un proceso continuo que reemplaza y sostiene la fuerza de trabajo y el tejido social (Gammage y Orozco, 2008). Es innegable que la teoría económica dominante ha marginalizado el proceso de reproducción de la fuerza de trabajo lo cual ha llevado a su invisibilización y a que haya sido relegado como un tema privado y femenino (Yáñez, 2009).

El modelo de trabajo basado históricamente en el modelo fordista-taylorista, se cimienta en el modelo androcéntrico, caracterizado por papeles claramente diferenciados entre hombres y mujeres, de modo que el hombre adquiere el papel de proveedor cuyo trabajo se centra en la esfera productiva y la mujer como la encargada de la esfera reproductiva, en labores domésticas y de cuidado.

Sin embargo, la producción de mercancías -bienes y servicios- requiere la puesta en marcha de energías, las cuales se gastan al igual que los otros insumos de producción y su recuperación, restitución y mejora son de interés no solo para los trabajadores y trabajadoras sino para el sistema económico, social y político como un todo (Yáñez, 2009).

Debido a las asimetrías persistentes entre las participaciones de hombres y mujeres en el hogar y el trabajo de reproducción, las mujeres son más propensas a tener conflicto entre su trabajo y el hogar (Mannheim y Schiffrin, 1984). De modo que como afirma Yañez, (2009:07):

"Incorporar la reproducción de la fuerza de trabajo como factor de la calidad del empleo remunerado implica evaluar el trabajo que se realiza dentro de la esfera laboral (empleo) según su capacidad de ser sostenedor en términos de reproducción de la fuerza de trabajo. Desde esta perspectiva, un trabajo productivo de calidad, esto es, un empleo 
decente, es aquel que es sustentable en términos de reproducción de la fuerza de trabajo"

\section{Medición de la calidad de empleo}

La conceptualización de los fenómenos es requisito previo para su medición (Gálvez, 2000). Las características de la noción de calidad del empleo mencionadas anteriormente, en especial la dualidad entre elementos subjetivos y objetivos genera dificultades a la hora de tratar de medirla; de modo que se trata de un constructo difícil de definir y operacionalizar dada su multidimensionalidad (Segurado y Agulló, 2002).

Aunque ha habido avances importantes en términos del desarrollo conceptual, la medición de la calidad de empleo o del trabajo decente todavía es un gran desafío. Definir indicadores - cuantitativos y cualitativos- que capturen las diversas dimensiones ha sido una tarea compleja y progresiva" (Weller y Roethlisberger, 2011).

La revisión que se presenta a continuación, se divide entre estudios que han planteado escalas de medición y las que han implementado o desarrollado otro tipo de índices e indicadores.

\subsection{Escalas de medición}

Las escalas de medición que han sido utilizadas comúnmente, por lo general cuentan con una serie de ítems o afirmaciones que deben ser calificadas por medio de valoración tipo Likert, que van desde 1 a 5 o desde 1 a 7 , variando entre completamente en desacuerdo hasta completamente de acuerdo. Perry, Champn, Conrad y Snyder (1995), para la medición de la calidad del empleo, en el caso específico de los maestros, utilizan una escala que considera los siguientes grandes ítems: Estrés, Carga de trabajo, Responsabilidad, Control sobre el trabajo, Sentido general positivo, Interés, Comunicación y el flujo de información, Complejidad y Diversidad.

Parasuraman, Purohit, Godshalk y Beutell (1996), tienen en cuenta la interdependencia del trabajo y de los roles familiares, para estos autores, es necesario examinar la importancia de las variables relacionadas tanto con la familia como con el trabajo para entender los determinantes del éxito y satisfacción profesional (Parasuraman, Greenhaus, y Granrose, 1992). En este caso, el 
género juega un papel importante, debido a que para las mujeres el conflicto entre trabajo y familia es más fuerte (Parasuraman, Purohit, Godshalk y Beutell ,1996). En el cuadro 2, se presentan las variables tenidas en cuenta por los autores para la medición de la CVL.

Cuadro 2. Variables para la medición de la CVL según Parasuraman et al., 1996

\begin{tabular}{|c|c|c|c|}
\hline \multicolumn{3}{|c|}{ DIMENSIÓN } & EXPLICACIÓN \\
\hline \multirow[t]{4}{*}{ Trabajo } & \multicolumn{2}{|c|}{ Autonomía } & $\begin{array}{l}\text { Grado de libertad en el trabajo y se asocia con } \\
\text { mayor satisfacción laboral y menor sensación de } \\
\text { estrés. }\end{array}$ \\
\hline & \multicolumn{2}{|c|}{ Inflexibilidad de horarios } & Fuente de conflicto entre el trabajo y la familia. \\
\hline & \multicolumn{2}{|c|}{ Sobre carga de trabajo } & $\begin{array}{l}\text { Magnitud perciba de trabajo y el sentimiento que } \\
\text { hay muchas cosas por hacer en poco tiempo } \\
\text { (Caplan et al., 1975). }\end{array}$ \\
\hline & \multicolumn{2}{|c|}{ Implicación laboral } & $\begin{array}{l}\text { Contexto psicológico de la importancia del trabajo } \\
\text { para el individuo e influencia la cantidad de } \\
\text { energía que se invierte en el trabajo (Greenhaus y } \\
\text { Beutell, 1985) }\end{array}$ \\
\hline \multirow[t]{4}{*}{ Familia } & \multicolumn{2}{|c|}{ Demandas paternales } & $\begin{array}{l}\text { Tener hijos aumenta la responsabilidad del cuidado } \\
\text { en el hogar, por tanto la existencia de niños en el } \\
\text { hogar y sus edades aumentan el conflicto entre } \\
\text { trabajo y familia. }\end{array}$ \\
\hline & \multirow{2}{*}{$\begin{array}{l}\text { Apoyo } \\
\text { del } \\
\text { cónyug } \\
\text { e }\end{array}$} & $\begin{array}{l}\text { Apoyo } \\
\text { instrumental }\end{array}$ & $\begin{array}{l}\text { Ayuda tangible por parte del compañero } \\
\text { sentimental en forma de participación en el } \\
\text { mantenimiento del hogar y el cuidado de los hijos. }\end{array}$ \\
\hline & & $\begin{array}{l}\text { Apoyo emocional } \\
\text { y de información }\end{array}$ & $\begin{array}{l}\text { Información, asesoramiento, demostración de } \\
\text { afecto y preocupación por el bienestar por parte del } \\
\text { cónyuge. }\end{array}$ \\
\hline & \multicolumn{2}{|c|}{ Implicación familiar } & Importancia que tiene la familia para el individuo. \\
\hline
\end{tabular}

Fuente: Parasuraman et al., 1996

Mientras tanto, Chiang y Krausse (2008), proponen cuatro indicadores para la medición de la CVL, los cuales se relacionan en el cuadro 3:

Cuadro 3. Variables para la medición de la CVL según Chiang y Krausse (2008)

\begin{tabular}{|l|l|}
\hline \multicolumn{1}{|c|}{ DIMENSIÓN } & \multicolumn{1}{c|}{ VARIABLES } \\
\hline Indicadores & Satisfacción con el ambiente físico del trabajo. \\
\cline { 2 - 2 } individuales & $\begin{array}{l}\text { Satisfacción con el reconocimiento que recibe de las } \\
\text { autoridades por su esfuerzo y trabajo }\end{array}$ \\
\hline
\end{tabular}




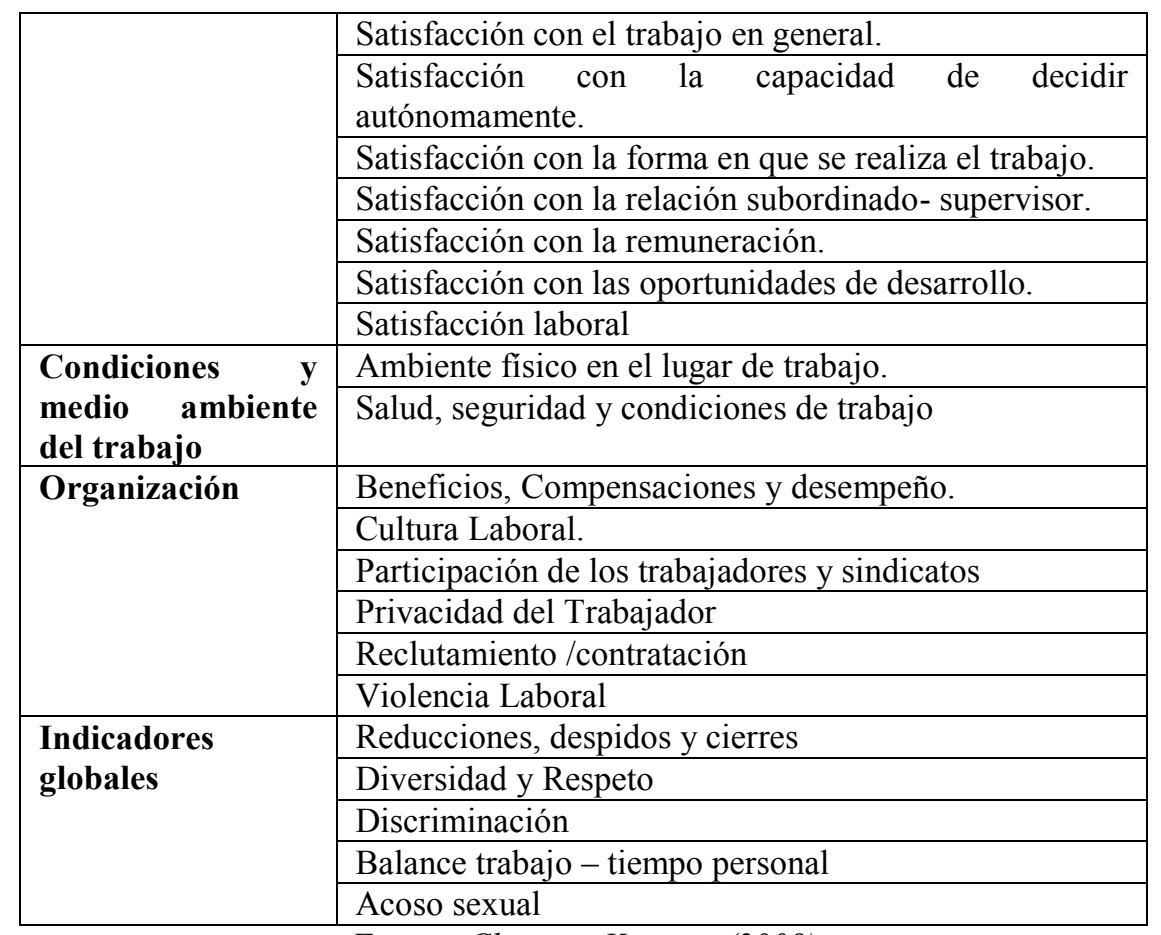

Fuente: Chiang y Krausse (2008)

Por su parte, Gómez (2010), elaboró un instrumento para la medición de la CVL, se trata de una escala de 74 ítems que relacionan 7 dimensiones, a saber, Soporte institucional para el trabajo, Seguridad en el trabajo, Integración al puesto de trabajo, Satisfacción por el trabajo, Bienestar logrado a través del trabajo, Desarrollo personal y Administración del tiempo libre. Dicha escala es denominada CVT-GOHISALO y ha sido usada en varios estudios (Gómez, 2010; Delgado, Inzulza y Delgado, 2012; Bonilla, Franco y Trinidad, 2012).

De otro lado Aziz et al. (2011) dividen las variables entre trabajo y no trabajo y encuentran que tanto las unas como las otras tienen influencia en la determinación de la calidad de vida laboral. Para la medición de estas variables usan escalas tipo Likert de 1 a 5 . Siguiendo a Parasuraman et al., (1996) toman las siguientes variables:

Cuadro 4. Variables para la medición de la CVL según Azziz et al., 2011

\begin{tabular}{|l|l|}
\hline \multicolumn{2}{|c|}{ VARIABLES } \\
\hline Relacionadas con el trabajo & Contexto del trabajo \\
\hline
\end{tabular}




\begin{tabular}{|l|l|}
\hline & Conflicto de rol de trabajo \\
\cline { 2 - 2 } & Tiempo de trabajo \\
\cline { 2 - 2 } & Inflexibilidad de horarios \\
\hline Relacionadas con la familia & Contexto familiar \\
\cline { 2 - 2 } & Conflicto familiar \\
\hline
\end{tabular}

Fuente: Azziz et al., 2011

Asimismo, el instituto Quality of Working Life del Reino Unido (QOWL, 2013), diseña y ajusta constantemente escalas para la medición de la calidad del trabajo. La escala denominada "Work-Related Quality of Life" (WRQoL) materializa una extensa investigación realizada acerca del tema, además, es una medida psicométrica de la calidad de la vida laboral de un empleado, plenamente probada y completa. Las dimensiones que relaciona la WRQoL se mencionan a continuación:

Cuadro 5. Variables para la medición de la CVL según WRQoL (2013)

\begin{tabular}{|l|l|}
\hline \multicolumn{1}{|c|}{ FACTOR } & \multicolumn{1}{c|}{ ¿QUÉ MIDE? } \\
\hline $\begin{array}{l}\text { Carrera y satisfacción } \\
\text { en el trabajo }\end{array}$ & Satisfacción general con el trabajo y con el desarrollo de la carrera \\
\hline $\begin{array}{l}\text { Condiciones } \\
\text { Trabajo }\end{array}$ & $\begin{array}{l}\text { Ambiente físico de trabajo y condiciones. } \\
\text { Tener las herramientas y equipos adecuados para realizar el trabajo }\end{array}$ \\
\hline Bienestar General & Bienestar psicológico y físico, felicidad. \\
\hline $\begin{array}{l}\text { Interconexión trabajo- } \\
\text { hogar }\end{array}$ & $\begin{array}{l}\text { ¿En qué medida la organización entiende y trata de ayudar las } \\
\text { presiones fuera del trabajo? } \\
\text { Flexibilidad organizativa. }\end{array}$ \\
\hline Estrés en el trabajo & $\begin{array}{l}\text { Niveles de estrés relacionados con el trabajo. } \\
\text { Grado en que las presiones y demandas son aceptables y no } \\
\text { excesivos o "estresantes". }\end{array}$ \\
\hline Control en el Trabajo & Participación en la toma de decisiones \\
\hline
\end{tabular}

Fuente: QOWL (2013)

Además de las escalas de medición, otros enfoques se han basado en índices e indicadores para determinar la CVL. Se destacan Farné (2003); Mauro, Medel y Yánez, (2009) y Weller y Rothlisberger (2011).

De acuerdo con Posso (2010), uno de los trabajos más destacados en Colombia al respecto de la medición y el análisis de la CVL, es el de Farné (2003). Este autor calculó 
un índice de calidad del empleo (ICE) para Colombia, utilizando la metodología de Rodgers y Reinecke (1998).

Usó cuatro variables: ingreso, modalidad de contratación, afiliación a la seguridad social y horario de trabajo. Teniendo en cuenta que un nivel aceptable del índice se puede considerar de sesenta puntos, para Colombia, el comportamiento fue precario, debido a que el índice arrojó un valor de 37,5\% para el conjunto de las trece áreas metropolitanas en 2001.

Asimismo, se calculó el índice para los asalariados y para los trabajadores independientes. Para los primeros el índice fue de $44 \%$ y para los trabajadores independientes, 27,5\%. Finalmente, comparó los resultados del índice entre Colombia y Chile, encontrando que Chile se encuentra en mejor posición que Colombia, ya que en Chile los puestos buenos y malos son minoritarios, y por la concentración se genera en empleos con condiciones intermedias (Rodgers y Reinecke, 1998), mientras que en Colombia los buenos empleos son muy pocos y el deterioro se extiende a una gran cantidad de empleos.

Por su parte, en el estudio realizado por Mauro et al. (2009), se usaron técnicas tanto cuantitativas (encuesta) como cualitativas (talleres de validación) con el objetivo de destacar las condiciones de trabajo sobre las que actúa la nueva organización del trabajo y como dichas condiciones afectan la calidad del trabajo y vida de los trabajadores y trabajadoras. Para tal fin, incluyeron seis dimensiones, las cuales se relacionan en el siguiente cuadro.

Cuadro 6. Variables para la medición de la CVL según Mauro et al., 2009

\begin{tabular}{|c|c|c|}
\hline \multicolumn{2}{|c|}{ DIMENSIÓN } & VARIABLE \\
\hline \multirow[t]{3}{*}{$\begin{array}{l}\text { Organización del } \\
\text { tiempo del trabajo } \\
\text { remunerado }\end{array}$} & $\begin{array}{l}\text { Extensión de la jornada de } \\
\text { trabajo y mecanismos } \\
\text { empresariales } \\
\text { prolongación de las jornadas. }\end{array}$ & $\begin{array}{llr}\text { Horas que } & \text { las } & \text { personas } \\
\text { dedican } & \text { al } & \text { trabajo } \\
\text { remunerado } & & \\
\end{array}$ \\
\hline & \multirow[t]{2}{*}{$\begin{array}{l}\text { Estrategias empresariales } \\
\text { para aumentar la extensión } \\
\text { de la jornada }\end{array}$} & $\begin{array}{ll}\text { Mecanismos } & \text { de } \\
\text { prolongación de horas de } \\
\text { trabajo }\end{array}$ \\
\hline & & Consecuencias de la \\
\hline
\end{tabular}




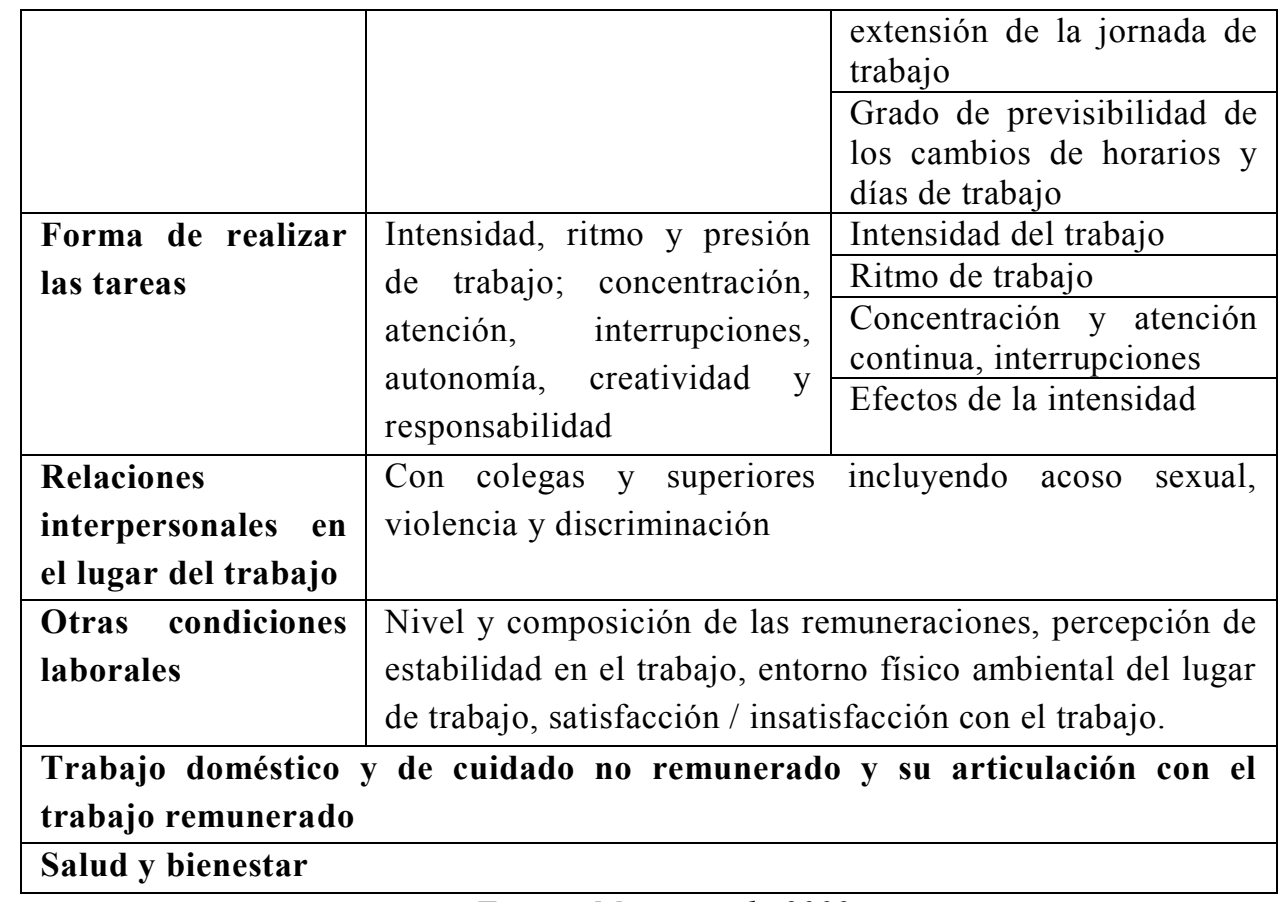

Fuente: Mauro et al., 2009

Continuando con los estudios realizados para la medición de la CVL, en el cuadro 7 se presentan las variables usadas por los Weller y Rothlisberger (2011), para América Latina.

Cuadro 7. Variables para la medición de la CVL según Weller y Rothlisberger, 2011

\begin{tabular}{|l|l|}
\hline \multicolumn{2}{|c|}{ INDICADORES } \\
\hline \multirow{2}{*}{ Ingresos } & Salarios \\
\cline { 2 - 2 } $\begin{array}{l}\text { Estabilidad de } \\
\text { trabajo } \begin{array}{l}\text { Beneficios no salariales } \\
\text { ingresos }\end{array}\end{array}$ & Tipos de contrato \\
\cline { 2 - 2 } $\begin{array}{l}\text { Protección socio- } \\
\text { laboral }\end{array}$ & Tipo de pago \\
\cline { 2 - 2 } & Salud \\
\cline { 2 - 2 } & Maternidad \\
\cline { 2 - 2 } & Jubilación \\
\cline { 2 - 2 } & Discapacidad \\
\cline { 2 - 2 } & Desempleo \\
\cline { 2 - 2 } & Igual pago para igual trabajo \\
\hline \multirow{4}{*}{$\begin{array}{l}\text { Organización del } \\
\text { trabajo }\end{array}$} & Númplimiento de legislación \\
\cline { 2 - 2 } & Intensidad del trabajo \\
\hline
\end{tabular}




\begin{tabular}{|l|l|}
\hline \multirow{4}{*}{$\begin{array}{l}\text { Integración } \\
\text { social }\end{array}$} & Riesgos ocupacionales (Salud, Accidentes) \\
\cline { 2 - 2 } & Ambiente físico (contaminación, ruido) \\
\cline { 2 - 2 } & Interés del trabajo (monotonía) \\
\cline { 2 - 2 } & Participación en decisiones \\
\cline { 2 - 2 } & $\begin{array}{l}\text { Organización de intereses laborales } \\
\text { acoso) }\end{array}$ \\
\hline $\begin{array}{l}\text { Desarrollo social del trabajo (con interacciones, trato digno, libre de } \\
\text { personal }\end{array}$ & Capacitación \\
\cline { 2 - 2 } & Perspectiva de trayectoria ascendente \\
\hline
\end{tabular}

Fuente: Weller y Rothlisberger, 2011

El anterior conjunto de indicadores se encuentra esquematizado de la siguiente forma:

Figura 1. Factores que determinan la calidad del empleo

\begin{tabular}{|l|l|l|}
\hline Aspectos económicos & Aspectos de calidad & Aspectos institucionales \\
\hline \begin{tabular}{|l|l|}
\hline Contexto productivo \\
(productividad y \\
heterogeneidad)
\end{tabular} & $\begin{array}{l}\text { Caracteristicas de la mano } \\
\text { de obra (capital humano) }\end{array}$ \\
\hline $\begin{array}{l}\text { Otros factores de producción } \\
\text { (capital, tecnologia, etc.) } \\
\text { Organización del proceso } \\
\text { productivo }\end{array}$ & $\begin{array}{l}\text { Calidad del puesto } \\
\text { de trabajo }\end{array}$ & $\begin{array}{l}\text { Normas sobre } \\
\text { seguridad, salud, } \\
\text { higiene en el trabajo }\end{array}$ \\
\hline
\end{tabular}

Fuente: Weller y Rothlisberger, 2011

\section{Modelo de medición de la calidad de empleo con enfoque de género}

Como afirma Farné (2003), para realizar un estudio adecuado de calidad de empleo se deben tener en cuenta tres elementos: proporcionar una definición adecuada del fenómeno, definir las variables determinantes y proponer indicadores para su medición.

Para el presente caso se usará un conjunto de indicadores. Considerando que el trabajo de Weller y Rothlisberger (2011) es uno de los más actuales, que ve la CVL desde un punto de vista holístico y que fue diseñado para América Latina; la medición de la CVL propuesta aquí, 
se basará en este trabajo, incorporando ajustes con base en los elementos subjetivos / objetivos y de la reproducción de la fuerza de trabajo discutidos anteriormente. En el cuadro, se presentan las variables a tener en cuenta.

Cuadro 8. Variables para la medición de la CVL

\begin{tabular}{|c|c|}
\hline \multicolumn{2}{|r|}{ DIMENSIONES } \\
\hline \multirow[t]{3}{*}{ Ingresos } & Salarios \\
\hline & Pago oportuno \\
\hline & Beneficios no salariales \\
\hline \multirow{2}{*}{$\begin{array}{l}\text { Estabilidad de } \\
\text { trabajo e ingresos }\end{array}$} & Tipos de contrato \\
\hline & Tipo de pago \\
\hline \multirow{6}{*}{$\begin{array}{l}\text { Protección socio- } \\
\text { laboral }\end{array}$} & Salud \\
\hline & Maternidad-Paternidad \\
\hline & Jubilación \\
\hline & Discapacidad \\
\hline & Igual pago para igual trabajo \\
\hline & Cumplimiento de legislación \\
\hline \multirow{5}{*}{$\begin{array}{l}\text { Organización del } \\
\text { trabajo }\end{array}$} & Número y organización de horas de trabajo \\
\hline & Intensidad del trabajo \\
\hline & Riesgos ocupacionales (Salud, Accidentes) \\
\hline & Ambiente físico (contaminación, ruido) \\
\hline & Interés del trabajo (monotonía) \\
\hline \multirow[t]{3}{*}{ Integración social } & Participación en decisiones \\
\hline & Autonomía \\
\hline & $\begin{array}{l}\text { Ambiente social del trabajo (con interacciones, trato digno, libre de } \\
\text { acoso) }\end{array}$ \\
\hline \multirow[t]{2}{*}{ Desarrollo personal } & Capacitación \\
\hline & Perspectiva de trayectoria ascendente \\
\hline $\begin{array}{l}\text { Trabajo } \\
\text { reproductivo }\end{array}$ & Horas dedicadas al trabajo y a la familia \\
\hline Satisfacción & Satisfacción general con el trabajo \\
\hline
\end{tabular}

Debido a que hay preguntas que se pueden aplicar directamente a la empresa, el cuestionario se divide en dos, uno para la organización y otro para el individuo. En el anexo 1, se presenta el instrumento de medición para el individuo y en el anexo 2, el instrumento para la empresa, lo anterior, además, para contrastar el punto de vista de los empleadores y los empleados. 


\section{Reflexiones finales}

La calidad de vida laboral ha sido un tema ampliamente estudiado, especialmente a partir de los años 60, debido a sus implicaciones, por un lado para el bienestar de las personas y por otro en la productividad de las empresas. Los enfoques que ha tenido la noción de calidad de vida laboral, han evolucionado a través del tiempo y tienen múltiples dimensiones.

En un principio se asoció a la CVL únicamente con elementos relacionados con el trabajo, es decir, se limitaba a las variables que provenían exclusivamente del medio laboral. Sin embargo, posteriores estudios demostraron que debido a la interdependencia entre el trabajo y los roles familiares, para entender los determinantes de la satisfacción profesional era indispensable tener en cuenta las variables relacionadas con la familia (Parasuraman, et al., 1992; Parasuraman, et al.., 1996; Aziz et al., 2011).

Un instrumento que intente medir la CVL, teniendo en cuenta el enfoque de género, debe incorporar dos elementos, la relación entre las características objetivas y subjetivas de las dimensiones del empleo y el papel de la reproducción de la fuerza de trabajo. 


\section{Anexos}

\section{ANEXO 1 \\ Instrumento de medición para los individuos}

Sexo

Edad

Estado civil

$\mathrm{N}^{\circ}$ de hijos o personas a cargo

Unidad

Antigüedad en la unidad

Tiempo que lleva trabajando

Ciudad donde trabaja

Ciudad donde vive

Nivel educativo

¿Cuál es su salario por hora?

¿Recibe su salario de forma oportuna?

¿Tiene algún tipo de reconocimiento no salarial? ¿Cuál?

¿Qué tipo de contrato tiene?

¿Qué tipo de pago recibo?

¿Ha tenido algún problema de salud ocupacional en el último año?

$\mathrm{Si}$ es padre/madre iha recibido los reconocimientos deley por parte de la empresa?

Si tiene alguna discapacidad ¿la empresa tiene alguna facilidad para usted?

Las remuneraciones en la empresa dependen del trabajo realizado

¿En la empresa se cumple la legislación vigente?

El número y la organización delas horas de trabajo está de acuerdo a sus capacidades

Existe políticas de riesgos ocupacionales

¿Tiene alguna participación en las decisiones dela empresa?

¿Tiene en las decisiones de su puesto de trabajo?

¿Recibe capacitaciones por parte de la empresa?

¿En la empresa hay ascensos?

¿Cuántas horas dedica al trabajo remunerado?

¿Cuántas horas dedica al trabajo no remunerado?

¿Cuántas horas dedica a las labores del hogar? 
¿HASTA QUE PUNTO ESTÁ DE ACUERDO CON LO SIGUIENTE?

Me siento satisfecho/a con mi salario

Me siento satisfecho/a con la forma de pago

Me siento satisfecho/a con el reconocimiento que recibo de las autoridades por su esfuerzo y trabajo

Me siento satisfecho/a con mi contrato

Me siento satisfecho/a con la estabilidad laboral

Siento que mi trabajo me genera problemas de salud

Me siento satisfecho/a con el trato dado por parte de la empresa durante y después de mi matemidad/patemidad

Siento que puedojubilarme correctamente trabajando en esta empresa

Si tiene alguna discapacidad ¿Me siento satisfecho/a con las facilidades que me presta la empresa para realizar bien mi trabajo?

¿Siente que en la empresa se da igual pago para igual trabajo?

Me siento satisfecho/a con el cumplimiento dela legislación dentro de la empresa

Me siento satisfecho/a con el número y la organización de las horas de trabajo

Me siento sobrecargado con el trabajo

Me siento satisfecho/a con el manejo de los riesgos ocupacionales en la empresa

Siento que existe contaminación excesiva en mi ambiente de trabajo

Siento que existe ruido excesivo en mi ambiente de trabajo

Siento que mi trabajo es monótono

Me siento satisfecho/a conla participación en las decisiones en mi ambiente de trabajo Me siento satisfecho/a conla capacidad de decidir autónomamente.

Me siento satisfecho/a con las interacciones sociales de mi trabajo

Me siento satisfecho/a con el trato en mi trabajo

Alguna vez me he sentido acosado/a en mi trabajo

Me siento satisfecho/a con las oportunidades de desarrollo y ascenso dentro de la empresa

Siento que el tiempo que dedico al trabajo es justo

Siento que el tiempo que dedico al trabajo no remunerado es justo

Siento que existe equidad en las horas que los miembros del hogar dedican a las labores del mismo

Me siento satisfecho/a con mi trabajo

\begin{tabular}{|c|c|c|c|c|}
\hline \multirow[t]{2}{*}{$\begin{array}{l}\text { Muy en } \\
\text { desacuerdo }\end{array}$} & \multicolumn{3}{|c|}{ Neer- } & \multirow[t]{2}{*}{$\begin{array}{l}\text { May de } \\
\text { acuerdo }\end{array}$} \\
\hline & $\begin{array}{l}\text { En } \\
\text { esacuerdo }\end{array}$ & & $\begin{array}{c}\text { De } \\
\text { ccuerdo }\end{array}$ & \\
\hline 1 & 2 & 3 & 4 & 5 \\
\hline 1 & 2 & 3 & 4 & 5 \\
\hline 1 & 2 & 3 & 4 & 5 \\
\hline 1 & 2 & 3 & 4 & 5 \\
\hline 1 & 2 & 3 & 4 & 5 \\
\hline 1 & 2 & 3 & 4 & 5 \\
\hline 1 & 2 & 3 & 4 & 5 \\
\hline 1 & 2 & 3 & 4 & 5 \\
\hline 1 & 2 & 3 & 4 & 5 \\
\hline 1 & 2 & 3 & 4 & 5 \\
\hline 1 & 2 & 3 & 4 & 5 \\
\hline 1 & 2 & 3 & 4 & 5 \\
\hline 1 & 2 & 3 & 4 & 5 \\
\hline 1 & 2 & 3 & 4 & 5 \\
\hline 1 & 2 & 3 & 4 & 5 \\
\hline 1 & 2 & 3 & 4 & 5 \\
\hline 1 & 2 & 3 & 4 & 5 \\
\hline 1 & 2 & 3 & 4 & 5 \\
\hline 1 & 2 & 3 & 4 & 5 \\
\hline 1 & 2 & 3 & 4 & 5 \\
\hline 1 & 2 & 3 & 4 & 5 \\
\hline 1 & 2 & 3 & 4 & 5 \\
\hline 1 & 2 & 3 & 4 & 5 \\
\hline 1 & 2 & 3 & 4 & 5 \\
\hline 1 & 2 & 3 & 4 & 5 \\
\hline 1 & 2 & 3 & 4 & 5 \\
\hline 1 & 2 & 3 & 4 & 5 \\
\hline
\end{tabular}




\section{ANEXO 2 \\ Instrumento de medición para la empresa}

Tamaño de la empresa

Tiempo en el mercado

¿En la empresa se dan los respectivos reconocimientos de matemidad/patemidad?

¿Se ha jubilado algún empleado?

¿En la empresa se dan las respectivas facilidades para las personas discapacitadas?

¿En la empresa se da igual pago para igual trabajo?

¿En la empresa se cumple la legislación vigente?

La intensidad del trabajo

Existe políticas de riesgos ocupacionales

¿Existe contaminación excesiva en el ambiente de trabajo?

¿Existe ruido excesivo en el ambiente de trabajo?

¿Los trabajos son monótonos?

¿Los empleados tienen algún tipo de participación en las decisiones

de la empresa?

¿Los empleados tienen la capacidad de decidir autónomamente?

¿Los empleados pueden hacer interacciones sociales?

¿Los empleados reciben capacitaciones por parte de la empresa?

¿Los empleados tienen la oportunidad de ascender en la empresa? 


\section{BIBLIOGRAFÍA}

- Alderfer, Clayton (1977): “Group and intergroup relations”. En Improving Life at Work, de J. R. Hackman y J. L. Suttle, editado por J. R. Hackman y J. L. Suttle. Goodyear, Santa Monica, California.

- Arvey, Richard D; Itzak, Harpaz y Hui, Liao (2004): “Work Centrality and Post-Award Work Behavior of Lottery Winners”. En The Journal of Psychology, n 5, pp. 404-420.

- Aziz, Rafidah Abdul; Nadzar, Fuziah Mohd; Husaini, Haslinda; Maarof, Asmah; Mohd Radzi, Salleh y Izhairi, Ismail (2011): "Quality of work life of librarians in government academic libraries in the Klang Valley, Malaysia”. En The International Information \& Library Review, no 43 pp. 149-158.

- Becker, Gary (1987): Tratado sobre la familia. España: Alianza Editorial.

- Bonilla, Carmen Patricia; Adalberto Franco, Sergio y Trinidad, Ana Silvia (2012): “Calidad de vida laboral de conductores de pasaje foráneo". En Revista Cubana de Salud y Trabajo, no 2, pp. 11-16.

- Caplan, Robert D; Cobb, Sidney; French Jr, John R P; Harrison, R. Van y Pineau Jr, S. R. (1975): Job demands and worker health. NIOSH Report.

- Che Rose, Raduan; LooSee Beh, Jegak Uli y Khairuddin, Idris (2006): “Quality Of Work Life: Implications Of Career Dimensions”. En Journal of Social Sciences, n² 2, pp. 61-67.

- Chiang, María Margarita y Ayres Krausse, Karina (2008): "Estudio empírico de calidad de vida laboral, cuatro indicadores: satisfacción laboral, condiciones y medioambiente del trabajo, organización e indicador global, sectores privado y público: Desarrollo, aplicación y validación del instrumento". En Horizontes empresariales. Concepción, Chile: Universidad del Bio-Bio, pp. 23-50.

- Clark, Andrew E. y Andrew J. Oswald (1994): “Unhappiness and unemployment”. En The Economic Journal, no 424 , pp. 648-659.

- Cunningham, Barton y Ted Eberle (1990): “A guide to job enrichment and redesign”. En Personnel, no 2, pp. 56-61. 
- Delamotte, Yves y Shin-ichi, Takezawa (1984): Quality of working life in international perspective. Geneva: International Labour Office.

- Delgado, Diemen; Inzulza, Miguel y Delgado, Fabián (2012): “Calidad de vida en el trabajo: Profesionales de la salud de Clínica Río Blanco y Centro de Especialidades Médicas”. En Medicina y Seguridad del Trabajo, nº 228, pp. 216-223.

- Downing, S; Ryan, G; McNeive, A; Mariani, M. y Parlangeli, O (1995): "The Quality of Working Life Concept”. En Symbiosis of Human and Artifac, pp.1011-1016.

- Escribano, Francisco y Pardo, Isabel. (2010): “Mujer e investigación en economía”. En Mujeres y Economía, no 852 , pp. 127-137.

- Farné, Stefano (2003): Estudio sobre la calidad del empleo en Colombia. Oficina Internacional del trabajo. Oficina Regional para América Latina y el Caribe.

- Flacso (2010): La construcción social del género y la economía. Facultad Latinoamericana de Ciencias Sociales.

- Frey, Bruno S. y Stutzer, Alois (2002): "What Can Economists Learn from Happiness Research?”. En Journal of Economic Literature, no 2, pp. 402-435.

- Galić, Zvonimir y Plećaš, Mara (2012): “Quality of Working Life during the Recession: The Case of Croatia". En Croatian Economic Survey, no 1, pp. 5-41.

- Gálvez, Thelma (2000): Medición de la calidad y precariedad del empleo. Chile, pp. 317325.

- Gammage, Sarah y Orozco, Mónica (2008): "El trabajo productivo no remunerado dentro del hogar: Guatemala y México”. En Serie: estudios y perspectivas. México: CEPAL, Unidad de Desarrollo Social.

- Gandelman, Néstor y Piani, Giorgina (2013): "Quality of Life Satisfaction among Workers and Non-Workers in Uruguay”. En Soc Indic Res, n 111, pp. 97-115.

- Gómez, María Alejandra (2010): “Calidad de vida laboral en empleados temporales del Valle de Aburrá - Colombia”. En Revista Ciencias Estratégicas, n² 24, pp. 225-236.

- Greenhaus, Jeffrey H. y Beutell, Nicholas J. (1985): "Sources of conflict between work and family roles". En The Academy of Management Review, n 1, pp. 76-88. 
- Greenhaus, Jeffrey; Bedeian, Arthur G. y Mossholder, Kevin (1987): "Work experiences, job performances and feelings of personal and family well-being”. En Journal of Vocational Behaviour, no 2, pp. 200-215.

- Guzmán, Virginia y Todaro, Rosalba (2002):. “Apuntes sobre género en la economía global". En Centro de Estudios de la Mujer.

- Index Mundi (2012): Mundo Perfil Población 2012.

- Ingelgard, Anders y Norrgren, Flemming (2001): "Effects of change strategy and topmanagement involvement on quality of working life and economic results". En International Journal of Industrial Ergonomics, nº 27, pp. 93-105.

- Mannheim, Bilha y Schiffrin, Meira (1984): "Family structure, job characteristics, rewards and strains as related to work-role centrality of employed and self-employed professional women with children”. En Journal of Organizational Behavior, n 2, pp. 83-101.

- Martínez, Julia (2011): Androcentrismo en la ciencia económica: mitos, misoginias y machismos. Departamento de Economía. El Salvador: UCA.

- Mauro, Amalia; Medel, Julia y Yáñez, Sonia (2009):. "Calidad del trabajo y género. Evidencias cuantitativas y cualitativas”. En Cuadernos de Investigación. ¿Malos tiempos para un "buen” trabajo? Calidad del trabajo y género. no 4, pp. 11-28.

- Nadler, David A; y Lawler, Edward E. (1983): "Quality of Work Life: Perspectives and Directions in Organisational Dynamics”. En Organizational Dynamics, n 3, pp. 20-30.

- Nicholson, Nigel, (1995) (ed.): The Blackwell Encyclopedia of Management and Encyclopedic Dictionaries, The Blackwell Encyclopedic Dictionary of Organizational Behavior. Oxford: Blackwell Publishers Ltd.

- Noor, Sarina Muhamad y Abdullah, Mohamad Adli (2012): “Quality Work Life among Factory Workers in Malaysia”. En Procedia - Social and Behavioral Sciences, pp. 739 - 745.

- Nussbaum, Martha, y Sen, Amartya (1996): La calidad de vida. México D.F.: Fondo de Cultura Económica.

- OIT (2013): El Programa de Trabajo Decente. 
- Parasuraman, Saroj; Greenhaus, Jeffrey H. y Granrose, Cherlyn Skromme (1992): "Role Stressors, Social Support, and Well-Being Among Two-Career Couples". En Journal of Organizational Behavior, $\mathrm{n}^{\circ}$ 4, pp. 339-356.

- Parasuraman, Saroj; Purohit, Yasmin S; Godshalk, Veronica M. y Beutell, Nicholas J. (1996): "Work and Family Variables, Entrepreneurial Career Success, and Psychological WellBeing”. En Journal of Vocational Behavior, n 3, pp. 275-300.

- Pautassi, Laura C. (2007): El cuidado como cuestión social desde un enfoque de derechos. Mujer y desarrollo. Santiago de Chile: CEPAL.

- Perry, Patricia D; Chapman, David W. y Snyder Jr, Conrad W. (1995): “Quality of teacher worklife and classroom practices in Botswana". En International Journal of Educational Development, $\mathrm{n}^{\mathrm{o}} 2$, pp. 115-125.

- Pichler, Florian y Wallace, Claire (2009): "What are the Reasons for Differences in Job Satisfaction across Europe? Individual, Compositional, and Institutional Explanations”. En European Sociological Review, no 4, pp. 535-549.

- Pineda, Javier A. (2006): Calidad del empleo e inequidades de género. Bogotá: Consejería Presidencial para la Mujer y la Equidad de Género.

- PNUD (2011): Sostenibilidad y Equidad: Un mejor futuro para todos. Informe sobre Desarrollo Humano. Nueva York: Programa de las Naciones Unidas para el Desarrollo.

- Posso, Christian Manuel (2010): “Calidad del empleo y segmentación laboral: un análisis para el mercado laboral colombiano 2001-2006”. En Desarrollo y sociedad, pp. 191-234.

- QOWL (2013): "Work-Related Quality of Life scale"

- Queralt, Laura y Ruiz, Ana Rosa (2003): “Perspectiva de género como eje transversal del quehacer académico e institucional: El caso del instituto técnico de Costa Rica”. En Colección de Materiales digitales para la docencia. Centro de Desarrollo Académico.

- Rodgers, G. y Reinecke G. (1998): “La calidad del empleo: perspectivas y ejemplos de Chile y Brasil". En Reestructuración, integración y mercado laboral. Crecimiento y calidad del empleo en economías abiertas. OIT. 
- Rodríguez, Corina (2010): “Análisis económico para la equidad: Los aportes de la economía feminista". En Consejo Nacional de Investigaciones Cientificas y Técnicas y Centro Interdisciplinario para el Estudio de Políticas Públicas, $\mathrm{n}^{\circ} 2$

- Saklani, Ruchi (2004): "Quality of work life in the Indian context: An empirical investigation". En Decision, nº 2, pp. 101-135.

- Sanchís, Norma (2005): "Los mapas del comercio: Una mirada sobre las geografías cambiantes de América Latina". En Capítulo Latinoamericano . Red Internacional de Género y Comercio.

- Scanlan, Stephen (2004): "Women, Food Security, and Development in Less-Industrialized Societies: Contributions and Challenges for the New Century”. En World Development, n 11, pp. $1807-1829$.

- Segurado, Almudena y Agulló, Esteban (2002): “Calidad de vida laboral: hacia un enfoque integrador desde la Psicología Social”. En Psicothema, no 4, pp. 828-836.

- Sen, Amartya (1999): Alocución en la 87a reunión de la OIT, Conferencia Internacional del Trabajo. Trabajo decente como eje de la equidad: Una agenda en las políticas sociales. Editado por María Estela Lanari. Gibebra.

. (1987): “Gender and Co-operative Conflicts”. En Wider Working Papers. nº 18.

. (1990): Gender and Co-operative Conflicts Persistent Inequalities: Women and World Development. Editado por Irene Tinker. New York: Oxford University Press, 123-49.

- Serey, Timothy (2006): “Choosing a Robust Quality of Work Life”. En Business Forum, n' 2, pp. 7-10.

- Solsona, Núria (1996): "La voz de las mujeres en la ciencia de los siglos XVII y XVIII". En Ingenium, pp. 125-136.

- Spector, Paul (2008): Industrial and organizational behavior. Hoboken: NJ: John Wiley and Sons.

- Spencer, David A. (2012): "Promoting High Quality Work: Obstacles and Opportunities". En Journal of Business Ethics, no 3, pp. 583-597. 
- Straw, Ronnie J. y Heckscher, Charles C. (1984): "QWL: New working relationships in the communication industry". En Labor Studies Journal, n 3, pp. 261-74.

- Suttle, Lloyd (1977): "Improving Life at Work Problems and Perspectives" En Improving Life at Work, de J. R. Hackman y J. L. Suttle, editado por J. R. Hackman y J. L. Suttle. Goodyear, Santa Monica, California.

- Walton, R. E. (1973): “Quality of working life: what is it?”. En Sloan Management Review, $\mathrm{n}^{\mathrm{o}} 1, \mathrm{pp} .11-21$.

- Weller, Jürgen y Roethlisberger, Claudia (2011): "La calidad del empleo en América Latina”. En Serie macroeconomía del desarrollo. no 110 . Santiago de Chile,: Naciones Unidas. CEPAL. División de Desarrollo Económico.

- Winkelmann, Liliana y Winkelmann, Rainer (1998): "Why are unemployed so unhappy? Evidence from panel data". En Economica, pp. 1-15.

- Yáñez, Sonia (2009): “Cambios del trabajo y nuevas exigencias de la reproducción de la fuerza de trabajo: Perspectivas analíticas para el estudio de la calidad del empleo con enfoque de género". En ¿Malos tiempos para un “buen” trabajo? Calidad del trabajo y género. Santiago de Chile: Centro de estudios de la mujer, pp. 7-10. 